\title{
OBTENCIÓN DE ETANOL A PARTIR DE RESIDUOS DE PODA, MEDIANTE HIDRÓLISIS ÁCIDA E HIDRÓLISIS ENZIMÁTICA
}

\section{ETHANOL FROM WASTE OF PRUNING BY ACID HYDROLYSIS AND ENZYMATIC HYDROLYSIS}

\author{
LeslyTejeda' ${ }^{1}$ Julio Quintana², Jairo Pérez ${ }^{3}$, Harold Young ${ }^{4}$
}

\begin{abstract}
${ }^{1}$ Ingeniera Química, Magíster en Ingeniería Ambiental, Docente investigadora, programas ambientales - Fundación Universitaria Tecnológico Comfenalco - Cartagena. Cartagena, Bolívar (Colombia). Bario España, Cra. 44D No. 30ª-91. lptbenitez@gmail. com. ${ }^{2}$ Ingeniero Ambiental, auxiliar de investigación, programas ambientales - Fundación Universitaria Tecnológico Comfenalco - Cartagena. Cartagena, Bolívar (Colombia). Bario España,Cra. 44D No. 30ª-91.jquintana.chacon@hotmail.com. ${ }^{3}$ Ingeniero Ambiental, auxiliar de investigación, programas ambientales - Fundación Universitaria Tecnológico Comfenalco - Cartagena. Cartagena, Bolívar (Colombia). Bario España,Cra. 44D No 30ª-91. jairo_perez15@hotmail.com ${ }^{4}$ Ingeniero Ambiental, auxiliar de investigación, programas ambientales - Fundación Universitaria Tecnológico Comfenalco - Cartagena. Cartagena, Bolívar (Colombia). Bario España,Cra. 44D No 30a-91. haroldyoung2003@hotmail.com
\end{abstract}

Rev. U.D.C.A Act. E Div. Cient. 14(1): 111 - 116, 2011

\section{RESUMEN}

Enestainvestigación, sepretendió estudiar el aprovechamiento de los residuos de poda y la búsqueda de materias primas para producir biocombustibles. Se llevó a cabo la hidrólisis de la biomasa lignocelulósica contenida en los residuos de poda, de la Fundación Universitaria Tecnológico Comfenalco, con el fin de producir jarabes glucosados para, potencialmente, ser convertidos en bioetanol. Se aplicaron dos metodologías: la hidrólisis ácida, usando ácido sulfúrico diluido y la hidrólisis enzimática, con enzimas celulasas. A manera de resultados, se obtuvo una mayor conversión con la hidrólisis ácida de todas las muestras (entre 10 y $30 \mathrm{~g} / \mathrm{L}$ de glucosa) que con la hidrólisis enzimática de los residuos mezclados $(8,83 \mathrm{~g} / \mathrm{L}$ de glucosa), quedando abierta la posibilidad de obtener biocombustibles a partir de este tipo de residuos. Se hace necesaria la optimización de los procesos con el propósito de mejorar el rendimiento en la producción de glucosa.

Palabras clave: Etanol, hidrólisis, jarabes glucosados, residuos lignocelulósicos.

\section{SUMMARY}

This research pretended to evaluate the use of yard pruning residues and to look for raw materials to produce bio-fuels. The hydrolysis of lignocellulosic biomass contained in the waste of the tree trimming at the Fundación Universitaria Tecnológico Comfenalco was carried out to produce glucose syrups to, potentially, be converted into bio-ethanol. Two methods were employed, the acid hydrolysis using dilute sulfuric acid and the enzymatic hydrolysis using cellulose enzymes. As a result, a higher conversion of all samples was obtained with the acid hydrolysis (between 10 to $30 \mathrm{~g} / \mathrm{L}$ of glucose) as with the enzymatic hydrolysis of mixed waste $(8.83 \mathrm{~g} / \mathrm{L}$ of glucose), suggesting that bio-fuels can be obtained, beginning from this type of vegetal residues. It is necessary to optimize the process in order to improve the output in the glucose production.

Key words: Ethanol, hydrolysis, glucose syrups, lignocellulosic wastes.

\section{INTRODUCCIÓN}

La Fundación Universitaria Tecnológico Comfenalco cuenta con extensas zonas verdes, las cuales, demandan mantenimiento y servicios, generando residuos de podas, que deben ser tratados por personal de la fundación y, posteriormente, por una empresa de aseo, generando costos por su transporte y su manipulación. Cuello et al. (2008) estimaron que en la institución, mensualmente, se producen $19,2 \mathrm{~kg}$ de estos residuos. Los residuos de poda de jardín contienen altos porcentajes de biomasa lignocelulósica, que se puede usar para obtener bioetanol, previa conversión a glucosa (Sun E Cheng, 2002). Dada la acogida que han tenido los biocombustibles en los últimos años es necesario explorar nuevas materias primas adecuadas para su 
obtención sostenible y que no pongan en peligro la seguridad alimentaria. La conversión de material lignocelulósico en glucosa, se puede hacer por vía ácida o por vía enzimática y ha sido ampliamente documentada.

López-Miranda et al. (2009) probaron los métodos de pre-tratamiento alcalino, ácido y explosión con vapor, para efectuarla hidrólisis enzimática de aserrín de pino y recuperar los azúcares contenidos en él. Encontraron que el pre-tratamiento con $\mathrm{NaOH}$ a $120^{\circ} \mathrm{C}$ proporcionó mejores resultados que los realizados con ácido sulfúrico diluido y por explosión con vapor a $121^{\circ} \mathrm{C}$. Se obtuvieron concentraciones de azúcares reductores de $48 \%$, después de un pre-tratamiento, con $8 \%$ de $\mathrm{NaOH}$, durante 85 minutos, a $120^{\circ} \mathrm{C}$, a un $\mathrm{pH}$ de hidrólisis de 4,5 , durante $240 \mathrm{~h}$, con una carga enzimática de $25 \mathrm{UPF} / g$ de carbohidratos totales.

Lis et al. (2000) llevaron a cabo la hidrólisis ácida de paja usando ácido clorhídrico y ácido sulfúrico; Ferrer et al. (2002) obtuvieron jarabes glucosados en la hidrólisis ácida del bagacillo de la caña de azúcar y Aguilar Rivera $\&$ Canizales Leal (2004) estudiaron la hidrólisis ácida de la cascarilla de cebada y hallaron una conversión del $70,83 \%$ a $110^{\circ} \mathrm{C}$, en un tiempo de 210 minutos, con una concentración de ácido sulfúrico del $1 \%$.

Boutros et al. (2005) efectuaron la hidrólisis ácida de bagazo utilizando ácido sulfúrico; Moya et al. (2007) realizaron la hidrólisis ácida de residuos de poda de olivo empleando ácido fosfórico, mientras que Lázaro \& Arauzo (1994) manipularon la hidrólisis enzimática de residuos de alcachofa y de espárrago y Gamez et al. (2006) hidrolizaron muestras de bagazo de caña de azúcar con ácido fosfórico al $4 \%$ a $122^{\circ} \mathrm{C}$, durante 300 minutos y obtuvieron concentraciones de 17,6g/L de xilosa, 2,6g/L de arabinosa y 3,0g/L de glucosa.

A nivel nacional, Monsalve et al. (2006) obtuvieron jarabe glucosado, a partir de la hidrólisis ácida de la cáscara de banano y Tejeda et al. $(2009,2010)$ experimentaron la hidrólisis ácida de cáscaras de naranja, piña, mango y papaya. En el país, se ha estudiado poco la hidrólisis ácida o enzimática de residuos de poda.

En este trabajo, se analizó la hidrólisis por vía ácida y enzimática de los residuos de jardín, con el fin de obtener jarabes glucosados para su posterior conversión en etanol.

\section{MATERIALES Y MÉTODOS}

Para el desarrollo de la investigación, se identificaron tres tipos de residuos de poda en la institución: tallos, hojas y frutos. Estos fueron recolectados en sacos, con capacidad de 10L; posteriormente, la reducción de tamaño, se llevó a cabo moliendo los residuos. La materia prima fue caracterizada por cromatografía líquida de alta resolución, para determinar los contenidos de celulosa, hemicelulosa, lignocelulosa y glucosa iniciales.

Para la experimentación, se tomaron muestras de $15 \mathrm{~g}$ de cada residuo, para la hidrólisis ácida y una muestra de $20 \mathrm{~g}$, que contenía los residuos mezclados en idénticas proporciones, para la hidrólisis enzimática.

El primer paso consistió en la eliminación de lignina, proceso, en el cual, se sumergieron las muestras en una solución de $\mathrm{NaOH} 0,1 \mathrm{~N}$; a los 15 minutos, se adicionó 0,816g de sulfato de calcio y se dejó en reposo por tres horas y, por último, se separó el material particulado de la solución. El hidróxido de sodio degrada la lignina y el sulfato de calcio evita la degradación de la celulosa causada por el hidróxido de sodio. La lignina disuelta fue eliminada de las muestras por decantación y el sólido que contenía la celulosa y la hemicelulosa fue conservado para la hidrólisis.

La hidrólisis ácida, se llevó a cabo en Erlenmeyer de 250mL, donde se mezcló una solución de ácido sulfúrico $\left(\mathrm{H}_{2} \mathrm{SO}_{4}\right)$ concentrado, el cual, se diluyó al $5 \%$ y se añadió $25 \mathrm{~mL}$ a cada $50 \mathrm{~g}$ de muestra. Posteriormente, ésta solución adicionada a las muestras, se llevó a un esterilizador eléctrico de presión de vapor (autoclave),manteniendo una presión y una temperatura fija de $1 \mathrm{~atm}(15 \mathrm{psi})$ y $125^{\circ} \mathrm{C}$, respectivamente, durante 15 minutos; luego, las muestras fueron sometidas a centrifugación para separar el jarabe glucosado.

La hidrólisis enzimática, se llevó a cabo en bio-reactor utilizando las enzimas celulasas y considerando las condiciones adecuadas de humedad y $\mathrm{pH}$ para su mantenimiento.

\section{RESULTADOS Y DISCUSIÓN}

\section{Caracterización de residuos}

La caracterización de los residuos de jardín permitió conocer la composición inicial de los mismos para tener un estimativo de la producción de glucosa, mediante los dos mecanismos de hidrólisis. El porcentaje de glucosa en los residuos proporciona el valor inicial y con el porcentaje de celulosa, se puede establecer el potencial de producción de glucosa. La tabla 1 muestra los resultados de la determinación.

La muestra que presentó mayor contenido de glucosa fue la de las frutas y el mayor contenido de celulosa, se registró en los tallos.

La literatura reporta un contenido promedio de glucosa en algunas flores, variadas entre el 31 y el 34\% (Salamanca, 1999) y un contenido promedio de celulosa entre 10 y $30 \%$ (Delfín Alcalá et al. 2003). 
Tabla 1. Caracterización de residuos.

\begin{tabular}{|l|c|c|c|}
\hline \multicolumn{1}{|c|}{ Muestra } & Tallos & Hojas & Flores \\
\hline Glucosa, g/L & 3,84 & 13,04 & 20,31 \\
\hline Hemicelulosa, g/L & 15,42 & 27,60 & 23,15 \\
\hline Celulosa, g/L & 42,54 & 28,67 & 24,17 \\
\hline Promedio, g/L & 20,60 & 23,10 & 23,66 \\
\hline
\end{tabular}

Los resultados del contenido de celulosa obtenidos para tallos y para hojas, se hallan muy cerca a lo reportado por Delfín Alcalá et al. (2003), quienes percibieron32\%, para las hojas y $36 \%$, para los tallos.

Se puede concluir que los residuos de poda contienen glucosa, celulosa y hemicelulosa, por lo cual, son potencialmente aprovechables en la producción de jarabes glucosados, por vía ácida o enzimática, con un mayor contenido por parte de los residuos de tallo, al poseer mayor contenido de celulosa y menor de hemicelulosa. La glucosa contenida en estos residuos es susceptible de ser utilizada bien sea para la obtención de bioetanol, a través de la fermentación alcohólica o en la producción de otros materiales de importancia industrial, como el ácido láctico, a través de una fermentación láctica.

\section{Hidrólisis ácida}

Los jarabes glucosados obtenidos a partir de la hidrólisis ácida de los residuos previamente separados por hojas, tallos y flores, fueron evaluados para determinar el contenido de glucosa final y el rendimiento en la conversión, resultado que se muestra en la tabla 2.

De acuerdo a estos valores, se puede observar que, en general, la conversión de celulosa a glucosa es baja, con excepción de las flores. El contenido de glucosa en los jarabes obtenidos a partir de los tallos y las hojas presentó un contenido de glucosa más bajo que los hallados en los jarabes elaborados a partir de las flores. Este resultado se esperaba, dado que las flores contienen alto contenido de glucosa, incluso, antes de su hidrólisis.

En todos los casos hubo un aumento del contenido de glucosa con respecto al contenido inicial, con lo cual, se puede verificar que parte de la celulosa contenida en los residuos se convirtió, químicamente, en glucosa.

Al comparar estos resultados con otras investigaciones relacionadas con la hidrólisis ácida de los residuos lignocelulósicos, se observa un comportamiento similar.
Tabla 2. Resultados jarabes glucosados hidrólisis ácida.

\begin{tabular}{|c|c|c|c|}
\hline $\begin{array}{c}\text { Generación de } \\
\text { glucosa }\end{array}$ & Tallos & Hojas & Flores \\
\hline Glucosa inicial $(\mathrm{g} / \mathrm{L})$ & 3,84 & 13,04 & 30 \\
\hline Glucosa final (g/L) & 10,77 & 16,85 & 30,46 \\
\hline Glucosa producida (g/L) & 6,93 & 3,81 & 0,46 \\
\hline \% Rendimiento & $16 \%$ & $13 \%$ & $2 \%$ \\
\hline
\end{tabular}

Lis et al. (2000) hidrolizaron paja aplicando, como agentes hidrolíticos, ácido clorhídrico y ácido sulfúrico y obtuvieron jarabes con máximo 12g/L de contenido de glucosa; Monsalve et al. (2006) produjo jarabe glucosado, a partir de la hidrólisis ácida de la cáscara de banano, con un contenido de glucosa de $20 \mathrm{~g} / \mathrm{L}$ y una conversión del $15 \%$; Ferrer et al. (2002) lograron un 22\% de conversión en la hidrólisis ácida del bagacillo de la caña de azúcar; Aguilar Rivera E Canizales Leal (2004) realizaron el estudio cinético de la hidrólisis ácida de la cascarilla de cebada y hallaron conversiones entre 23 y $59 \%$, trabajando con diferentes concentraciones de ácido sulfúrico y prolongando el tiempo de reacción; Boutros et al. (2005), en la hidrólisis ácida del bagazo manejando ácido sulfúrico concentrado en una sola etapa y en una etapa modificada, consiguió conversiones entre el 50 y el $88 \%$ y, finalmente, Moya et al. (2007), ejecutó la hidrólisis ácida de residuos de poda de olivo usando ácido fosfórico, alcanzando conversiones entre el 10 y el $20 \%$.

Teóricamente y teniendo en cuenta el contenido inicial de glucosa y celulosa disponible en los residuos de jardín, se puede obtener un jarabe con concentración más alta; sin embargo, al realizar la eliminación de lignina, posiblemente, se pierden azúcares solubles y es probable que, parte de la celulosa, se solubilice en el mismo procedimiento.

Otra forma de incrementar la conversión es a través del pre-tratamiento, para el cual, se tienen diferentes opciones y combinación de ellas, como: explosión con vapor sin catalizador, calentamiento en agua caliente líquida, calentamiento en agua caliente con $\mathrm{pH}$ controlado, flujo de agua caliente, ácido diluido, flujo de ácido, explosión con amonio y cal, entre otros (Cardona Alzate et al. 2005).

\section{Hidrólisis enzimática}

La tabla 3 muestra los resultados del nivel de glucosa logrado en los jarabes glucosados, producidos por la hidrólisis enzimática de los residuos mezclados; el promedio de estos resultados y el porcentaje es calculado con base en la cantidad inicial de glucosa y celulosa. 
Tabla 3. Resultados de glucosa, hidrólisis enzimática.

\begin{tabular}{|c|c|}
\hline Jarabes & Niveles de glucosa $\mathbf{( g / L )}$ \\
\hline 1 & 9,4 \\
\hline 2 & 8,9 \\
\hline 3 & 8,2 \\
\hline PROMEDIO & 8,83 \\
\hline
\end{tabular}

De acuerdo con los resultados conseguidos y expuestos en la tabla 3, el contenido de glucosa en el jarabe elaborado a partir de los residuos de poda mezclados es menor que en todos los jarabes glucosados producidos por hidrólisis ácida e, incluso, es menor que el contenido de glucosa promedio en los residuos antes de su hidrólisis, con lo que se puede decir que no hubo una conversión significativa.

Con lo anterior, se confirmó que la hidrólisis ácida registra un mayor rendimiento que la hidrólisis enzimática.

Al comparar los resultados alcanzados en esta experimentación con los registrados por otros autores, se encuentran rendimientos parecidos en unos casos, como los siguientes: Lázaro E Arauzo (1994) efectuaron la hidrólisis enzimática de residuos de alcachofa y de espárrago realizando un tratamiento previo con $\mathrm{NaOH}$ y obtuvieron jarabes glucosados, con contenido de 1,7 y $4,5 \mathrm{~g} / \mathrm{L}$ de glucosa y rendimientos de reacción entre 3 y 10\%; Sánchez Villasclaras et al. (2003) estudiaron la influencia del pH en la hidrólisis enzimática de los residuos de poda de olivar y logró jarabes con un contenido máximo de $16 \mathrm{~g} / \mathrm{L}$ de glucosa. Otros autores mostraron mejores resultados en la hidrólisis enzimática, al optimizar las condiciones del pre-tratamiento, el tipo de enzimas y la concentración de las mismas.

Öhgren et al. (2007) alcanzaron altos rendimientos entre el 70 y el $74 \%$ en la producción de xilosa, que se forma por hidrólisis de la hemicelulosa, cuando emplearon enzimas xilanasas, para complementar a las enzimas celulasas, durante la hidrólisis enzimática de cáscaras de maíz; esto es favorable, porque la xilosa, al igual que la glucosa es un azúcar fermentable apta para ser convertida en etanol. Cardona Alzate et al. (2005) realizaron la sacarificación enzimática de la madera de Eucalyptus globulus, con miras a obtener bioetanol y lograron, en promedio, $26 \mathrm{mg}$ de glucosa/g de biomasa.

López Miranda et al. (2009) consiguieron jarabes glucosados por hidrólisis enzimática de aserrín de pino, con un rendimiento superior en la formación máxima de azúcares reductores del $48 \%$, aplicando un tratamiento previo, con $8 \%$ de $\mathrm{NaOH}$, durante 85 minutos, a $120^{\circ} \mathrm{C}$, a un pH de hidrólisis de 4,5 , durante $240 \mathrm{~h}$, con una carga enzimática de $25 \mathrm{UPF} / \mathrm{g}$ de carbohidratos totales.

Una causa del bajo rendimiento reportado durante la etapa de hidrólisis enzimática, se debe a que la glucosa y la celobiosa liberadas inhiben las enzimas implicadas en el proceso.

Otra posible causa de la baja conversión es que durante el pre-tratamiento se forman una serie de productos de degradación, que pueden afectar el rendimiento de la enzima, actuando como potenciales inhibidores, por lo tanto, es conveniente probar con diferentes técnicas de pretratamiento.

\section{Hidrólisis enzimática vs hidrólisis ácida}

La hidrólisis ácida mostró mayor rendimiento, alcanzando un 10,33\% de glucosa en comparación con la hidrólisis enzimática, que fue de $8,83 \%$, concluyendo que es más factible la producción de bioetanol usando ácidos como catalizadores.

En el cuadro 1, se presenta un comparativo que muestra las ventajas y las desventajas entre la hidrólisis catalizada con ácidos y la hidrólisis catalizada con enzimas.

Con el objetivo de optimizar el rendimiento en la producción de glucosa combinando las ventajas y las desventajas de una y otra forma de hidrólisis, algunos autores (Mejía et al. 2007) han propuesto un esquema combinado, en el cual, previo tratamiento térmico, se efectúa la hidrólisis ácida, seguida de la hidrólisis enzimática. Otra tecnología de creciente interés es la SFS (Sacarificación y Fermentación Simultáneas), con el fin de realizar, en un solo reactor, los pasos de hidrólisis enzimática y fermentación alcohólica. Es una técnica que aún se encuentra en estudio, debido a que para aplicarla es necesaria la modificación genética de los microorganismos para que puedan coexistir bajo las mismas condiciones los microorganismos responsables de la sacarificación y las levaduras fermentativas, responsables de la conversión de los azúcares en etanol.

Otros procesos usan agua en condiciones cercanas a las supercríticas, en las etapas de pre-tratamiento e hidrólisis, reduciendo estas etapas a una sola, con el fin de hidrolizar la celulosa a glucosa sin el uso de ácido y convertir este compuesto en etanol u otros productos de mayor valor económico (Cardona Alzate et al. 2005).

Si bien la producción de bioetanol es muy conveniente, debido a las grandes cantidades de residuos de podas existentes, la producción no ha resultado económica a escala industrial, a causa de los bajos rendimientos hallados en la hidrólisis ácida y enzimática, con vías a la obtención previa de glucosa. 
Cuadro 1.Hidrólisis enzimática vs hidrólisis ácida.

\begin{tabular}{|c|c|c|}
\hline \multirow{4}{*}{ 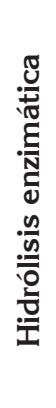 } & \multirow{2}{*}{$\begin{array}{l}\frac{n}{\tilde{J}} \\
\stackrel{0}{\tilde{J}} \\
\stackrel{0}{0}\end{array}$} & No requiere la neutralización del producto de la hidrólisis. \\
\hline & & La utilización de enzimas es ambientalmente más segura y sostenible que el uso de ácidos. \\
\hline & \multirow{2}{*}{ 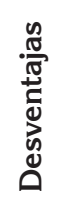 } & $\begin{array}{l}\text { La cristalinidad de las moléculas de celulosa y su asociación con la lignina, constituyen una verdad- } \\
\text { era barrera física a la penetración de las enzimas. }\end{array}$ \\
\hline & & $\begin{array}{l}\text { Se requiere un pre-tratamiento que permita que la celulosa esté disponible para la acción de las } \\
\text { enzimas celulolíticas. }\end{array}$ \\
\hline \multirow{4}{*}{ 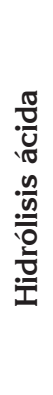 } & \multirow{2}{*}{ 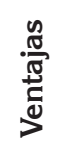 } & Se presentan rendimientos de reacción más altos. \\
\hline & & No requiere procesos de pre-tratamientos sofisticados. \\
\hline & \multirow{2}{*}{ 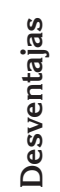 } & $\begin{array}{l}\text { La generación de productos de degradación, durante la hidrólisis ácida, puede afectar al microor- } \\
\text { ganismo encargado de realizar la posterior fermentación. }\end{array}$ \\
\hline & & Se hace necesario neutralizar los hidrolizados antes de la fermentación. \\
\hline
\end{tabular}

Agradecimientos: Los investigadores agradecen a la Fundación Universitaria Tecnológico Comfenalco y a la Corporación Autónoma Regional del Canal del Dique CARDIQUE- por facilitar el uso de sus laboratorios y asesorar la ejecución de la presente investigación. Conflictos de intereses: El manuscrito fue preparado y revisado con la participación de todos los autores, quienes declaramos que no existe ningún conflicto de intereses que ponga en riesgo la validez de los resultados presentados. Financiación: Este estudio fue financiado por la Fundación Universitaria Tecnológico Comfenalco, en modalidad Bolsa Concursable.

\section{BIBLIOGRAFÍA}

1. AGUILAR RIVERA, N.; CANIZALES LEAL, M.J. 2004. Cinética de la hidrólisis ácida de la cascarilla de cebada. Rev. Mex. Ing. Química. (México): 3(3):257263.

2. BOUTROS, F.; SARROUH, J.; JOVER, E. 2005.Estudio de la hidrólisis del bagazo con ácido sulfúrico concentrado utilizando dos variantes de una sola etapa y una sola etapa modificada para la obtención de etanol y análisis técnico-económico de dicho proceso. Ingeniería e Investigación (Colombia). 25(3):34-38.
3. CARDONA ALZATE, C.J.; SÁNCHEZ TORO, O.J.; MONTOYA RODRÍGUEZ, M.I.; QUINTERO SUÁREZ, J.A. 2005. Producción de etanol carburante: material lignocelulósico una nueva alternativa. Rev. Eidenar Univalle.3:47-55.

4. CUELlO, C.; GARCÍA, M.; RODRÍGUEZ, I.; DÍAZ MENDOZA, C. 2008. Formulación y diagnóstico del plan de Manejo Integral de Residuos. Revista Teknos. (Colombia). 3(1):19-23.

5. DELFÍN ALCALÁ, I.; DURÁN DE BAZUA, C. 2003. Biodegradación de residuos urbanos lignocelulósicos. Revista Internal Contaminación Ambiental. (México). 19(1):37-45.

6. FERRER, J.R.; PÁEZ, G.; ARENAS DE MORENO, L. 2002. Cinética de la hidrólisis ácida de bagacillo de caña de azúcar. U. Zulia. Revista Facultad de Agronomía (Venezuela). 19(1):23-33.

7. GAMEZ, S.; GONZÁLEZ, J.; RAMÍREZ, J.; GARROTE, G.; VÁSQUEZ, M. 2006. Study of the hydrolysis of sugar cane bagasse using phosphoric acid. J. Food Engineering. 74(1):78-88. 
8. LÁZARO, L.; ARAUZO, J.1994. Aprovechamiento de residuos de la industria de conservas vegetales: hidrólisis enzimática. Revista Zubía (España).12:227-240.

9. LIS, M.J.; CARRILLO, F.; COLOM, X.; MARTÍNEZ, D.; NOGUÉS, F. 2000. Hidrólisis ácida de paja previa a su tratamiento enzimático determinación de un modelo cinético. Ing. Química (España). 32(369):181-186.

10. LÓPEZ-MIRANDA, J.; SOTO-CRUZ, N.; RUTIAGAQUIÑONES, O. 2009. Optimización del proceso de obtención enzimática de azúcares fermentables a partir de aserrín de pino. Rev. Internal Contam. Ambient. (México): 25(2):95-102.

11. MEJÍA, L.F.; MARTÍNEZ CORREA, H.A.; BETANCOURT GUTIÉRREZ, J.E.; CASTILLÓN CASTAÑO, C.E. 2007. Aprovechamiento del residuo agroindustrial del mango común (Mangifera indica L.) en la obtención de azúcares fermentables. Ingeniería y Ciencia (Colombia). 3(6):41-62.

12. MONSALVE, G.J.; MEDINA DE PÉREZ, V.; RUÍZ COLORADO, A. 2006. Producción de etanol a partir de la cáscara de banano y de almidón de yuca. Dyna. (Colombia). 73(150):21-27.

13. MOYA, M.; ROMERO, I.; SÁNCHEZ, S.; RUÍZ, E.; CASTRO, A.; BRAVO V. 2007. Ethanolic fermentation of phosphoric acid hydrolysates from olive tree pruning. Industrial Crops and Products (España). 25(2):160-168.
14. ÖHGREN, K.; BURA, R.; SADDLER, J.; ZACCHI, G. 2007.Effect of hemicellulose and lignin removal on enzymatic hydrolysis of steam pretreated corn stover. Bioresource Techn. (Canada). 98(13):2503-2510.

15. SALAMANCA, G.1999. Determinación de azúcares mayoritarios en mieles de Apis mellifera por cromatografía líquida. O’apicultor, (Colombia). 29(7):17-18.

16. SUN, Y.; CHENG, J. 2002. Hydrolysis of lignocellulosic materials for ethanol production: A review. Bioresource Technology (USA). 83(1):1-11.

17. SÁNCHEZ VILLASCLARAS, S.; BRAVO RODRÍGUEZ, V.; GARCÍA MARTÍN, J.F. 2010. Producción de bioetanol a partir del residuo de la poda del olivo.Ed. Curbet Com. Gráfica, España. 92p.

18. TEJEDA, L.P.; TEJADA, C.; VILLABONA, A.; ALVEAR, M.R.; CASTILLO, C.R.; HENAO, D.L.; MARIMÓN W.; MADARIAGA, N.; TARÓN, A. 2010. Producción de bioetanol a partir de la fermentación alcohólica de jarabes glucosados derivados de cáscaras de naranja y piña. Rev. Educación en Ingeniería. 10:120-125.

19. TEJEDA, L.; TEJADA, C.; VILLABONA, A.; TARON, A.; ALVEAR, M.; CASTILLO, C.; HENAO, D.; MARIMON, W. 2009. Producción de etanol carburante a partir de las cáscara de mango (Mangifera indica) y papaya (Carica papaya). Ciencias e Ingeniería al Día. 4(1):4147.

Recibido: Julio 26 de 2010

Aceptado: Marzo 14 de 2011 